\title{
LUCES Y SOMBRAS DE LA CALIDAD DE LA DEMOCRACIA EN AMÉRICA LATINA
}

\author{
Manuel Alcántara Sáez*
}

\begin{abstract}
Resumen:
Se realiza en el artículo un estudio estadístico de la calidad de la democracia en América Latina, para lo cual se utiliza los índices de Freedom House, IDD (Fundación Honrad Adenauer), EIU (The Economist Intelligence Unit) y el elaborado por Levine Molina (2007), que ostentan una similitud altamente significativa, los cuales han constatando grandes diferencias en la calidad de la democracia a nivel de los países de América Latina.
\end{abstract}

Palabras Clave: Democracia - Freedom House - Desarrollo Democrático.

\begin{abstract}
A bstract:
A statistical study of the quality of the democracy in Latin America is made in the article, for which it is used the indices of Freedom House, IDD (Fundacio'n Honrad Adenauer), EIU (The Economist Intelligence Unit) and the elaborated by Levine Molina (2007), that shows a similarity highly significant, which are stating great differences in the quality of the democracy at the countries of Latin America.
\end{abstract}

Key words: Democracy - Freedom House - Dremocratic development

* Vicerrector de Relaciones Internacionales y Cooperación de la Universidad Salamanca de España y profesor de Ciencia Política en la misma Universidad. 
Manuel Alcántara Sáez - Luces y sombras de la calidad de la democracia en América Latina

El término de calidad de la democracia, que comienza a abrirse paso hace apenas una década ${ }^{1}$, tiene un carácter complejo al estar vinculado tanto a significados diferentes para el término decalidad deacuerdo con los sectores industriales y de mercadotecnia, como a visiones dispares del concepto de democracia. En efecto, la calidad puede estar vinculada a un procedimiento por el que un producto de calidad es el resultado de un proceso riguroso de acuerdo con un protocolo preciso, pero también lo está al contenido, es decir es inherente a las características estructurales de un producto, y final mente tienequever con el resultado medido por el grado de satisfacción del usuario². Así mismo hay una visión de la democracia donde se enfatiza más su capacidad de provocar la participación de la ciudadanía, de estimular debates y deliberación sobrelas opciones que enfrenta un país o una comunidad, de proteger los derechos de los individuos y grupos marginales frente a los grupos de poder, de promover la justicia social ${ }^{3}$. Esta perspectiva es diferente a la de una democracia configurada sobre los valores de libertad, igual dad política y el control sobre las políticas públicas y sus hacedores a través del funcionamiento legítimo y legal de instituciones estables. Esta segundo versión, próxima a la conceptualización deDahl, puedemedirseen términos desu calidad si se satisfacen ocho dimensiones. Cinco de ellas tienen carácter procedimental, son: el imperio de la ley, la participación, la competición, la responsabilidad vertical y la responsabilidad horizontal. Dos tienen carácter sustantivo: respecto a las libertades civiles y políticas y la implementación progresiva de mayor igualdad política (y subsiguientemente social y económica). Por último se encuentra la dimensión "responsiveness" que enlaza las dimensiones procedimentales con las substantivas proveyendo una base para medir cuántas más o menos políticas públicas (incluyendo leyes, instituciones y gastos) corresponden con las demandas de los ciudadanos según han sido agregadas a través del proceso político ${ }^{4}$

Empíricamente pueden considerarse varios índices que con metodologías diferentes abordan aspectos relativos al desempeño dela política intentando medir esos distintos grados de calidad dela democracia. Como a continuación seva a constatar todos estos análisis, cuya similitud es altamentesignificativa, han puesto sobradamente de manifiesto las grandes diferencias que se dan entre los países de A mérica Latina. Setrata delosíndices deFreedom H ouse, el más antiguo deellos, ID D (Fundación Konrad Adenauer), TheE conomist IntelligenceU nit (EIU) y el elaborado por Leviney Molina (2007).

El índice de Freedom H ouse se estableceanual mente sobrela base deopiniones subjetivas de expertos que evalúan el estado de la libertad global según la 
experimentan los individuos. Por consiguiente no se trata de una evaluación del rendimiento de los gobiernos per se sino delos derechos y delas libertades que gozan las personas. El índice cuyo propósito es evaluar el grado de libertad, entendida como oportunidad para actuar espontáneamente en una variedad de terrenos fuera del control del gobierno y de otros centros de dominio potencial, se traduce en una escal a de 1 a 7 con dos apartados bien diferenciados para los derechos políticos y las libertades civiles. Los derechos políticos capacitan a la gente para participar libremente en el proceso político, incluyendo el derecho a votar libremente por distintas al ternativas en el ecciones legítimas, competir por cargos públicos, incorporarse a partidos políticos y a organizaciones y elegir representantes que tengan un impacto decisivo sobre las políticas públicas y que sean responsables anteel el ectorado. Las li bertades civiles tienen que ver con las libertades de expresión y de creencia, los derechos de asociación, el estado de derecho y la autonomía personal sin interferencias desdeel Estado.

\section{Cuadro I. Índice de Freedom House 2007}

\begin{tabular}{|l|c|}
\hline País & Índice \\
\hline Chile & 1 \\
\hline Costa Rica & 1 \\
\hline Uruguay & 1 \\
\hline Panamá & 1,5 \\
\hline Argentina & 2 \\
\hline Brasil & 2 \\
\hline República Dominicana & 2 \\
\hline México & 2,5 \\
\hline El Salvador & 2,5 \\
\hline Perú & 2,5 \\
\hline Bolivia & 3 \\
\hline Colombia & 3 \\
\hline Ecuador & 3 \\
\hline Honduras & 3 \\
\hline Nicaragua & 3 \\
\hline Paraguay & 3 \\
\hline Guatemala & 3,5 \\
\hline Venezuela & 4 \\
\hline Cuba & 7 \\
\hline
\end{tabular}

Los datos se refieren al periodo comprendido entre el 01-12-05 y el 31-12-06 Valores medios de los índices de derechos políticos y de libertades civiles

Fuente: http:/ / www .freedomhouse.org/ template.cfm?page=365\&year $=2007$ 
Todos los países latinoamericanos analizados por Freedom H ouse, menos Cuba, son considerados dentro de la categoría de democracias el ectoral es aspecto que supone la satisfacción de los siguientes cuatro criterios: un sistema político competitivo y multipartidista; sufragio universal para todos Ios ciudadanos; elecciones periódicas competitivas llevadas a cabo bajo condiciones de voto secreto, seguridad razonable en el voto, ausencia de fraude electoral masivo y que los resultados sean representativos del deseo dela gente; final mente, acceso público significativo de los partidos políticos más importantes al el ectorado a través de los medios de comunicación y a través de fórmulas de campaña general mente abiertas. F reedom H ouse establece como "libres" a aquellos países cuyo índice se sitúan entre 1 y 2,5 de manera que algo más de la mitad de los países latinoamericanos considerados se encuentran en esta categoría; "semi libres" son aquellos en los que el índice se sitúa entre 3 y 5 ; en la categoría de "no libres" para valores comprendidos entre 5,5 y 7 solamente se encontraría Cuba.

\section{C uadro II. Índice de D esarrollo D emocrático en A mérica Latina 2007}

\begin{tabular}{|l|c|}
\hline País & Índice 2007 \\
\hline Chile & 10,360 \\
\hline Costa Rica & 9,706 \\
\hline Uruguay & 9,384 \\
\hline Panamá & 6,452 \\
\hline Argentina & 6,123 \\
\hline México & 5,566 \\
\hline Honduras & 4,780 \\
\hline Colombia & 4,778 \\
\hline Brasil & 4,582 \\
\hline Perú & 4,107 \\
\hline El Salvador & 3,967 \\
\hline Paraguay & 3,880 \\
\hline Guatemala & 3,502 \\
\hline Bolivia & 3,281 \\
\hline Ecuador & 3,206 \\
\hline República Dominicana & 2,900 \\
\hline Venezuela & 2,848 \\
\hline Nicaragua & 2,730 \\
\hline
\end{tabular}

Fuente: Konrad Adenauer y Polilat.

http:/ / www.idd-lat.org/ Edicion\%202006.htm 
El índice de desarrollo democrático (ID D $)^{5}$, que descarta a Cuba por su carácter no democrático, permite establecer cuatro grupos de países plenamente diferenciados de mayor, medio-alto, medio-bajo y menor desarrollo democrático. El primer grupo lo integran Chile, Costa Rica, Uruguay. El segundo Panamá, Argentina y México. El tercer grupo, de desarrollo democrático medio-bajo, está compuesto por Honduras, Colombia, Brasil, Perú, El Salvador y Paraguay. El de menor desarrollo está integrado por Guatemala, Bolivia, Ecuador, R. Dominicana, Venezuela y Nicaragua.

\section{Cuadro III. El índice de democracia de EIU de 2006}

\begin{tabular}{|l|c|c|c|c|c|c|}
\hline \multicolumn{1}{|c|}{ País $^{1}$} & $\begin{array}{c}\text { Procesos } \\
\text { electorales y } \\
\text { pluralismo }\end{array}$ & $\begin{array}{c}\text { Funciona- } \\
\text { miento del } \\
\text { gobierno }\end{array}$ & $\begin{array}{c}\text { Partici- } \\
\text { pación } \\
\text { política }\end{array}$ & $\begin{array}{c}\text { Cultura } \\
\text { Política }\end{array}$ & $\begin{array}{c}\text { Liberta- } \\
\text { des } \\
\text { civiles }\end{array}$ & $\begin{array}{c}\text { Ín dice } \\
\text { total }\end{array}$ \\
\hline Costa Rica (25) & 9,58 & 8,21 & 6,11 & 6,88 & 9,41 & $\mathbf{8 , 0 4}$ \\
\hline Uruguay (27) & 10,00 & 8,21 & 5,00 & 6,88 & 9,71 & $\mathbf{7 , 9 6}$ \\
\hline Chile (30) & 9,58 & 8,93 & $5,, 00$ & 6,25 & 9,71 & $\mathbf{7 , 8 9}$ \\
\hline Brasil (42) & 9,58 & 7,86 & 4,44 & 5,63 & 9,41 & $\mathbf{7 , 3 8}$ \\
\hline Panamá (44) & 9,58 & 7,14 & 5,56 & 5,63 & 8,82 & $\mathbf{7 , 3 5}$ \\
\hline México (53) & 8,75 & 6,07 & 5,00 & 5,00 & 8,53 & $\mathbf{6 , 6 7}$ \\
\hline Argentina (54) & 8,75 & 5,00 & 5,56 & 5,63 & 8,24 & $\mathbf{6 , 5 3}$ \\
\hline Colombia (67) & 9,17 & 4,36 & 5,00 & 4,38 & 9,12 & $\mathbf{6 , 4 0}$ \\
\hline Honduras (69) & 8,33 & 6,43 & 4,44 & 5,00 & 7,06 & $\mathbf{6 , 2 5}$ \\
\hline El Salvador (70) & 9,17 & 5,43 & 3,89 & 4,38 & 8,24 & $\mathbf{6 , 2 2}$ \\
\hline Paraguay (71) & 7,92 & 5,00 & 5,00 & 4,38 & 8,53 & $\mathbf{6 , 1 6}$ \\
\hline Rep. Dom. (74) & 9,17 & 4,29 & 3,33 & 5,63 & 8,24 & $\mathbf{6 , 1 3}$ \\
\hline Perú (75) & 8,75 & 3,29 & 5,56 & 5,00 & 7,94 & $\mathbf{6 , 1 1}$ \\
\hline Guatemala (77) & 8,75 & 6,79 & 2,78 & 4,38 & 7,65 & $\mathbf{6 , 0 7}$ \\
\hline Bolivia (81) & 8,33 & 5,71 & 4,44 & 3,75 & 7,85 & $\mathbf{5 , 9 8}$ \\
\hline Nicaragua (89) & 8,25 & 5,71 & 3,33 & 3,75 & 7,35 & $\mathbf{5 , 6 8}$ \\
\hline Ecuador (92) & 7,83 & 4,29 & 5,00 & 3,13 & 7,94 & $\mathbf{5 , 6 4}$ \\
\hline Venezuela (93) & 7,00 & 3,64 & 5,56 & 5,00 & 5,88 & $\mathbf{5 , 4 2}$ \\
\hline Cuba (124) & 1,75 & 4,64 & 3,89 & 4,38 & 2,94 & $\mathbf{3 , 5 2}$ \\
\hline
\end{tabular}

${ }^{1}$ Entre paréntesis lugar mundial

Fuente: The Economist Intelligence Unit's index of democracy. The World in 2007. Londres.

http:/ / www.economist.com/ media/ pdf/ DEMOCRACY_INDEX_2007_v3.pdf 
Manuel Alcántara Sáez - Luces y sombras de la calidad de la democracia en América Latina

El tercer índice considerado es el de democracia de EIU ${ }^{6}$ clasifica y agrupa en cuatro categorías a 167 países, tiene, por tanto, una característica muy relevante y es la de contextualizar a los países latinoamericanos en el panorama mundial. El primer grupo, de democracias plenas apenas si representan el diecisiete por ciento del total; el segundo grupo de democracias devaluadas son el treinta y dos por ciento; los regímenes híbridos que constituyen el tercer grupo son el dieciocho por ciento; y finalmente se encuentran los regímenes autoritarios que suponen el treinta y tres por ciento. Es decir, grosso modo, la mitad de los países del mundo considerados no tienen el carácter de democráticos según esta clasificación y un tercio son directamenteautoritarios.

Para diecinueve países de A mérica Latina abordados en dicho estudio la gran mayoría se sitúa entre los dos primeros grupos de democracias plenas y devaluadas: Costa Rica y Uruguay están en el primero de ellos, y trece países se encuentran en el segundo. Lo relevante es, por consiguiente, que solamente hay tres casos de regímenes híbridos (Nicaragua, Ecuador y Venezuela) y uno de régimen autoritario (Cuba). Las dos variables constitutivas del índice que provocan la menor calidad de la democracia en los países latinoamericanos sesitúan en el ámbito, muy complementario por otra parte, de la participación política y de la cultura política. Los trece países concebi dos como democracias devaluadas cuentan con un bajo rango en la expresión de una ciudadanía poco activa políticamente hablando, apática, muy desconfiada y ajena al debate político.

Finalmente, el índice de Levine y Molina ${ }^{7}$ parte de una definición de la cal idad de la democracia como la medida en que los ciudadanos participan informadamente en procesos de votación libres, imparciales y frecuentes; influyen en la toma de decisiones políticas; y exigen responsabilidad a los gobernantes, y por la medida en queéstos últimos son quienes efectivamente toman las decisiones y lo hacen respondiendo a la voluntad popular. Esta definición identifica cinco dimensiones de la calidad dela democracia que son consideradas individualmentey queseagregan conformand o el propio índice, se trata de la decisión el ectoral, la participación, la responsabilidad (accountability), la respuesta a la voluntad popular (responsiveness) y la soberanía. 


\section{Cuadro IV . Índice de calidad de la democracia en A mérica Latina 2005 de Levine y Molina}

\begin{tabular}{|l|c|c|c|c|c|c|}
\hline \multicolumn{1}{|c|}{ País } & $\begin{array}{c}\text { Decisión } \\
\text { Electoral }\end{array}$ & $\begin{array}{c}\text { Partici- } \\
\text { pación }\end{array}$ & $\begin{array}{c}\text { Responsa- } \\
\text { bilidad }\end{array}$ & $\begin{array}{c}\text { Respuesta } \\
\text { a la } \\
\text { voluntad } \\
\text { popular }\end{array}$ & Soberanía & $\begin{array}{c}\text { Calidad de } \\
\text { la } \\
\text { Calidad de }\end{array}$ \\
\hline Uruguay & 90.7 & 55.3 & 47.9 & 85 & 80.5 & $\mathbf{7 1 . 9}$ \\
\hline Costa Rica & 87.0 & 44.5 & 37.3 & 51 & 97 & $\mathbf{6 3 . 4}$ \\
\hline Chile & 88.3 & 45.1 & 42.3 & 65 & 75.5 & $\mathbf{6 3 . 2}$ \\
\hline Argentina & 74.8 & 54.7 & 27.3 & 67 & 89.5 & $\mathbf{6 2 . 7}$ \\
\hline México & 71.8 & 50.1 & 27.1 & 66 & 91.5 & $\mathbf{6 1 . 3}$ \\
\hline Panamá & 75.7 & 45.2 & 32.4 & 61 & 91.5 & $\mathbf{6 1 . 2}$ \\
\hline R. Dominic. & 72.4 & 48.0 & 32.7 & 67 & 79.5 & $\mathbf{5 9 . 9}$ \\
\hline Brasil & 81.4 & 56.8 & 23.9 & 67 & 60.5 & $\mathbf{5 7 . 9}$ \\
\hline Perú & 78.8 & 56.5 & 21.8 & 56 & 70 & $\mathbf{5 6 . 6}$ \\
\hline Bolivia & 73.9 & 52.2 & 21.8 & 50 & 75.5 & $\mathbf{5 4 . 7}$ \\
\hline Nicaragua & 62.9 & 46.8 & 17.3 & 60 & 79.5 & $\mathbf{5 3 . 3}$ \\
\hline Colombia & 61.2 & 46.8 & 30.7 & 62 & 65.5 & $\mathbf{5 3 . 2}$ \\
\hline El Salvador & 67.8 & 41.2 & 29.3 & 64 & 62 & $\mathbf{5 2 . 9}$ \\
\hline Parag uay & 57.9 & 44.2 & 28.1 & 57 & 77.5 & $\mathbf{5 2 . 9}$ \\
\hline Venezuela & 50.7 & 51.4 & 24.5 & 74 & 62 & $\mathbf{5 2 . 5}$ \\
\hline Honduras & 59.9 & 45.8 & 21.0 & 49 & 79.5 & $\mathbf{5 1 . 0}$ \\
\hline Ecuador & 62.2 & 49.4 & 18.3 & 48 & 51 & $\mathbf{4 5 . 8}$ \\
\hline Guatemala & 47.7 & 37.4 & 20.3 & 54 & 63.5 & $\mathbf{4 4 . 6}$ \\
\hline
\end{tabular}

Fuente: Levine y Molina (2007).

Los resultados de este índice permiten de nuevo constatar la escala diferenciadora dela cal idad de la democracia en los países latinoamericanos. A unque las distancias reflejan un continuo muy estrecho entre los valores de Costa Rica y de Honduras, dejándose en los extremos a Uruguay, en la cima de mayor calidad, y a Ecuador y Guatemala como polo de menor cal idad, los restantes países proyectan un grupo de calidad al ta compuesto por Costa Rica, Chile, A rgentina, México y Panamá, otro de calidad media integrado por R. Dominicana, Brasil, Perú y Bolivia, y un tercer de calidad baja en el que se da cabida a Nicaragua, Colombia, El Salvador, Paraguay, Venezuela y Honduras. 
Manuel Alcántara Sáez - Luces y sombras de la calidad de la democracia en América Latina

La relación entre los cuatro índices pone de manifiesto una clara identidad a la hora de señalar cual es son los países más aventajad os democráticamente hablando, así como los que ocupan lugares mucho más atrasadosy, además, identifica nítidamente las diferencias existentes en la escal a de manera que avala la tesis de la heterogeneidad regional.

El hecho de que estos índices se basen fundamentalmente en criterios tendentes a analizar la calidad de los procesos desde el estricto imperio de los mecanismos institucionales que articulan el juego político permiteintentar esbozar una serie de hi pótesis cuyo carácter exploratorio requiere de mayor análisis. Desdela perspectiva de la oferta se puede considerar la cal idad de los servicios gubernamentales. Desde la perspectiva más inclinada hacia la demanda, cuatro son los el ementos de índoleinstitucional vinculados todos ellos al carácter representativo de la democracia que pueden estar en la base interpretativa de los diferentes niveles de calidad de la democracia, y que vendrían ligados: a la operatividad electoral, el funcionamiento de los partidos políticos, las relaciones entre el Poder Ejecutivo y el Poder Legislativo y los procesos de descentralización. A todos ellos habría que añadir un factor metainstitucional quese referiría a la cal idad de los políticos como sostén explicativo de la calidad dela democracia.

Voy a desarrollar muy brevemente en las páginas siguientes al gunas ideas que pueden servir para la operacionalización de estos elementos y para intentar descomponer su peso en el esbozo de una senda explicativa.

La calidad del gobierno, en su condición de administración pública, no ha mejorado en la región en la última década. Los diferentes indicadores que miden su rendimiento muestran un nivel claramente inferior al de otras regiones en vías de desarrollo. A demás, ponen de relieve que no se trata tanto deal go debido a la cuantía del gasto público dedicado, cuyo monto es ciertamente escaso, sino de aspectos ligados a tener pendientela superación dela debilidad institucional. Esta se traduce en el mantenimiento defórmulas declientelismo y de patronazgo a la hora dela contratación y dela promoción del personal donde brillan por su ausencia los procesos de selección competitiva, neutra y por mérito, y se mantienen diferentes formas de desigual dad interna como sucede en el ámbito de la remuneración (sueldo distinto por trabajos similares) o dela promoción dela mujer. I gualmente se encuentran ausentes mecanismos de definición de una carrera de servicio público y otros de evaluación del rendimiento ${ }^{\text {. }}$ 
Manuel Alcántara Sáez - Luces y sombras de la calidad de la democracia en América Latina

La operatividad electoral quiero articularla en dos dimensiones. La primera es relativa a cuestiones estrictamente organizativas y procedimental es que tienen que ver con el estricto desarrollo del proceso. A spectos ligados a la confección y actualización del padrón quegarantice la efectiva participación, al establecimiento de los col egios y de las mesas electoral es para hacer más accesible el sufragio, a la puesta en marcha de procedimientos de recuento rápidos y fiables, en fin, a la existencia de mecanismos garantes de la reparación de la totalidad de los derechos de el ectores y de candidatos. La segunda se refiere a la satisfacción efectiva de las funciones de representación sobre la base de asegurar la inclusión de los distintos grupos proyectándose su presencia en la arena política en función de su tamaño y, a la vez, de posibilitar la conformación de mayorías que den estabilidad y faciliten la acción de gobierno.

Los partidos políticos mantienen la posición más baja en la tabla de confianza institucional regional, como otras instituciones representativas de la democracia liberal quelanguidecen por debajo de los 50 puntos en una escala de 0 a 100 (el valor delos partidos es 35, el sistema judicial 43 y el legislativo 44, frente a las Fuerzas Armadas con 60 o la Iglesia con 69) 9 ${ }^{9}$. Sin embargo, comparando esta baja marca de los partidos con la que se da en otros países y tomando en consideración que una mayoría de los latinoamericanos considera que la democracia es inviable sin su presencia ${ }^{10}$, en mi opinión y contrariamente a un extendido sentir, no debería ser tomado el punto del repudio delos latinoamericanos a los partidos como un ejesignificativo desu papel presente en la política regional. Es la oligarquización de los mismos el aspecto probablemente más relevante. Si bien es un aspecto general de cualquier partido, en América Latina tiene que ver con bajos niveles de institucionalización tanto de los sistemas de partidos, como de los propios partidos. El primer ámbito ha veni do siendo objeto de atención constatándose su relación con otros aspectos del sistema político ${ }^{11}$. El segundo se vincula a matices organizativos propios dela misma maquinaria del partido que pasan por su financiación, el reclutamiento desu personal y las vías desu promoción y profesionalización, y a otros derivados del entramado democrático en el que están insertos lo quelleva a replantearse los procesos de selección desus líderes y de elaboración de sus programas mediante canales de mayor o de menor participación y transparencia.

Las relaciones entreel Poder Ejecutivo y el Poder Legislativo componen un escenario que ha sido fruto de una abundante literatura acerca desu impacto 
Manuel Alcántara Sáez - Luces y sombras de la calidad de la democracia en América Latina

en el devenir de la democracia en América Latina ${ }^{12}$, un espacio político dominado por el presidencialismo. Sin embargo, lejos de contemplar el problema como algo derivado de una determinada arquitectura constitucional la evidencia empírica requiere de análisis más minuciosos desde la perspectiva de las funciones desempeñadas por uno y otro Poder del Estado y dela manera en queinteractúan. El Poder Legislativo en América Latina ha sido con frecuencia ninguneado como actor relevante del juego político y ello es de particular importancia en una región dondeel número de países en los queel Presidente cuenta con un apoyo mayoritario estable en el Congreso es minoritario. Por consiguiente, deben analizarse con cuidado las funciones de los Ejecutivos y de los Legislativos ${ }^{13}$, así como los mecanismos que pueden llegar a producir consensos amplios en los que se debetener en cuenta no sol o factores institucionales o de relación defuerza partidista existente, sino también el ementos de carácter más subjetivo ${ }^{14}$.

Los procesos de descentralización, desdela perspectiva dela representación, configuran un escenario donde pueden estrecharse los lazos entre representantes y representados en la medida es que se den tres circunstancias: se reduzca y desagregue el tamaño del universo político, se distribuyan los recursos materiales y simbólicos a lo largo de diferentes unidades de poder, y se definan mecanismos de democracia horizontal y de rendición de cuentas. No obstante, la incidencia de estos factores en la calidad de la democracia no es evidente. Los procesos de "devolución" no siempre conducen a iguales resultados en función del caso concreto en el que se producen. Hay, al menos cuatro eventualidades cuya presencia (o ausencia) puede afectar significativamente el resultado: se trata de de la presencia de una(s) élite(s) con fuerte capacidad de liderazgo; de la existencia de grupos con identidades diferenciadores muy marcadas; de una situación global de al ta desigual dad en términos sociales, económicos, o culturales; y de factores internacionales que catalicen el proceso. Los acontecimientos vividos en el mundo andino son un excelente taller de prueba para constatar en qué medida esta variable juega contra la calidad dela democracia, mientras que en Brasil o en M éxico el sentido dela relación es positiva.

Final mente la clase política es una variable independiente que desempeña un papel muy importante en el proceso global de calidad de un sistema democrático como se ha mostrado con énfasis muy recientemente ${ }^{15}$. La cal idad delos políticos es un concepto difícil deestablecer pero puedeintegrar 
Manuel Alcántara Sáez - Luces y sombras de la

calidad de la democracia en América Latina

en el mismo su experiencia en el seno del partido, su experiencia en el oficio público (como representante o como cargo ejecutivo con un nivel mínimo de responsabilidad) y su nivel educativo. Los datos de un reciente trabajo ${ }^{16}$ ponen de relieve que solo dos de las tres democracias consideradas más fuertes y con mayores niveles de calidad en América Latina, Chile y Uruguay se caracterizan también por tener una mayoría de diputados de calidad ${ }^{17}$. Democracias más débiles en la región andina y Centroamérica y/ o con puntuaciones de calidad democrática bajos como Guatemala, Ecuador o Bolivia, obtienen también puntuaciones bajas en la clasificación de calidad de sus legisladores. La calidad de los políticos es un apartado que requiere ser tenido en mayor consideración que la manifestada hasta el presente.

\footnotetext{
1 Sobre diferentes medidas de calidad democrática en la región latinoamericana puede consultarse A Itman y Pérez-Liñán (2002). Una aproximación teórica y empírica puedeverseen O’Donnell, Vargas Cullell e lazzetta (2004); también en diferentes trabajos en J ournal of D emocracy, Vol. 15, no 4, 2004 y más ampliado en Diamond y Morlino (2005).

2 Ver Diamond y Morlino (2004: 21).

3 Ver A maral y Stokes (2005: 11).

4 Ver Diamond y Morlino (2004: 22).

5 EI IDD está compuesto por indicadores quemiden los atributos dela democracia formal sobrela base de elecciones libres, sufragio universal y participación plena (dimensión I) y otros de la democracia real articulados en tres dimensiones: el respeto de los derechos políticos y libertades civiles (dimensión II), la calidad institucional y la eficiencia política (dimensión III) y el ejercicio de poder efectivo para gobernar (dimensión IV), escindida esta última en la capacidad para generar políticas que aseguren bienestar y, en segundo término, eficiencia económica. Son, por tanto, indicadores procedentes depercepciones subjetivas pero también de rendimi entos empíricamente cuantificables.

6 Esteíndicees resultado dela integración de cinco variables queson: los procesos el ectorales y el pluralismo; el funcionamiento del gobierno; la participación política; la cultura política y las libertades civiles.

7 Ver Levine y Molina (2007).

8 Ver Clements, Faircloth y Verhoevewn (2007: 18-21).

9 Ver Seligson (2007: 89).

1044 por ciento de los latinoamericanos, como promedio, están de acuerdo con la pregunta: “ ¿pude haber democracia sin partidos?", con casos excéntricos como los de Ecuador y Haití, con 50,5 por ciento y 62,2 por ciento, respectivamente. Ver Seligson (2007: 90).

${ }^{11}$ El trabajo seminal de Mainwaring y Scully (1995) debe en este sentido ser considerado. A mbos autores establecieron cuatro condiciones para que un sistema democrático de partidos estuviera institucionalizado: la estabilidad en las reglas y en la naturaleza de la competición interpartidista, la posesión de raíces estables en la sociedad de los principales partidos, las elecciones como ruta primaria para acceder al gobierno y la relevancia de las organizaciones de los partidos (Mainwaring y Scully, 1995: 5)).

12 Todavía Valenzuela (2004) ha argumentado sobre el componenteconflictivo del propio presidencialismo latinoamericano que se había cobrado la cabeza de una docena de presidentes en las últimas dos décadas. Argumento quevenía a coincidir con el elaborado por Fish (2006) aplicable para los países poscomunistas donde demuestra la vinculación existente entre la fuerza del Poder Legislativo y la consolidación democrática.

${ }^{13}$ Sobre estas últimas y su peso puede verse A Icántara, García Montero y Sánchez López (2005).
} 
Manuel Alcántara Sáez - Luces y sombras de la calidad de la democracia en América Latina

${ }^{14}$ Con una correlación de fuerzas muy similar, un entramado institucional idéntico y siendo del mismo partido que su predecesor en menos de un año de gobierno, Felipe Calderón ha establecido una agenda colaboradora con el Congreso mexicano superior a la que en seis años ll egó a definir Vicente Fox.

${ }^{15}$ Ver A lcántara (2006).

16 Ver Martínez Rosón (2006).

17 Concepto que alude a las habilidades personales del político y a su integridad (Martínez Rosón, 2006: 181). 